\title{
Using the Heterodyne Method to Measure Velocities on Shock Physics Experiments
}

O. T. Strand, T. L. Whitworth

August 15, 2007

15th APS Topical Conference on Shock Compression of Condensed Matter Waikoloa, HI, United States June 24, 2007 through June 29, 2007 
This document was prepared as an account of work sponsored by an agency of the United States Government. Neither the United States Government nor the University of California nor any of their employees, makes any warranty, express or implied, or assumes any legal liability or responsibility for the accuracy, completeness, or usefulness of any information, apparatus, product, or process disclosed, or represents that its use would not infringe privately owned rights. Reference herein to any specific commercial product, process, or service by trade name, trademark, manufacturer, or otherwise, does not necessarily constitute or imply its endorsement, recommendation, or favoring by the United States Government or the University of California. The views and opinions of authors expressed herein do not necessarily state or reflect those of the United States Government or the University of California, and shall not be used for advertising or product endorsement purposes. 


\title{
Using the Heterodyne Method to Measure Velocities on Shock Physics Experiments
}

\author{
Oliver T. Strand and Tony L. Whitworth \\ Lawrence Livermore National Laboratory \\ Livermore, CA, USA
}

\begin{abstract}
We developed a velocimeter system several years ago that uses the heterodyne method [1]. This system is assembled from commercially available components that were developed for the telecommunications industry. There are several advantages of this system over the traditional VISAR method that has made it increasingly popular. This system is compact, portable, and relatively inexpensive. The maximum velocity of this system is determined by the electrical bandwidth of the electronics and the digitizer sample rate. The maximum velocity for the system described here is over $5 \mathrm{~km} / \mathrm{s}$.
\end{abstract}

\section{INTRODUCTION}

Velocimetry is an important diagnostic for many shock physics experiments. The velocities involved are usually in the kilometer-per-second range, which dictates that some form of interferometry be used to make these measurements. The traditional diagnostic is the Velocity Interferometer System for Any Reflector (VISAR), which is a commercially available product [2]. Fabry-Perot-based systems are also used, but are less common [3]. This paper describes a third interferometer system to make these velocity measurements that is based upon the heterodyne principle of generating a beat frequency proportional to the velocity. The system design and basic principles are described along with an example of data that was obtained with this heterodyne velocimeter.

\section{BASIC PRINCIPLES}

The notion of generating a beat signal is well known. For a velocimeter based upon this concept, the light from a laser is split into two paths. One path of the laser light is sent to the moving surface in a manner that allows a portion of the reflected light, which is now Doppler shifted, to be collected and sent to the detector. The other path of the laser light is considered 
the reference signal, which is not Doppler shifted, and is sent directly to the detector. For the system described here, the laser wavelength $\lambda$ is $1550 \mathrm{~nm}$, which corresponds to a frequency $\mathrm{f}_{0}$ of approximately $2 \times 10^{14} \mathrm{~Hz}$. At a velocity of 1 kilometer-per-second and this wavelength, the fractional change in frequency $\delta \mathrm{f} / \mathrm{f}$ is on the order of $10^{-5}$. The detector "sees" two CW components from the original frequencies and also the difference between those two frequencies, which is the beat frequency:

$$
I_{b}=I_{0}+I_{d}+\sqrt{I_{0} I_{d}} \sin \left(2 \pi f_{b} t+\phi\right)
$$

where $I_{b}$ is the intensity of the beat signal, $\mathrm{I}_{0}$ is the intensity of the reference (nondoppler-shifted) component of the signal, $I_{d}$ is the Doppler-shifted component of the signal, $\mathrm{f}_{\mathrm{b}}$ is the beat frequency, $\mathrm{t}$ is time, and $\phi$ is the relative phase between the Doppler-shifted and non-doppler-shifted signals. The beat frequency $f_{b}$ is related to the velocity $\mathrm{v}$ by:

$$
f_{b}=2\left(\frac{v}{c}\right) f_{0}
$$

For the system described here with a laser wavelength of $1550 \mathrm{~nm}$, the beat frequency is $1.29 \mathrm{GHz}$ for a velocity of $1 \mathrm{~km} / \mathrm{s}$.

\section{DESCRIPTION OF SYSTEM}

The heterodyne system developed for our applications uses single mode fibers to deliver the laser light from the laser to the probes and from the probes to the detectors. The laser is a 2 -watt $\mathrm{CW}$ fiber laser with $1550 \mathrm{~nm}$ wavelength. The output of the laser is sent to a $1 \times 4$ fiber splitter that then feeds four probes (Figure 1). The output of each leg of the fiber splitter is connected to port \#1 of a 3-

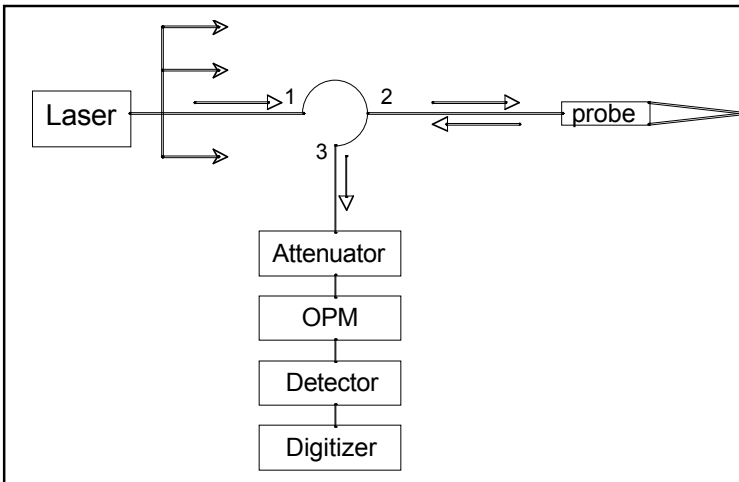

Figure 1 . The heterodyne system has 4 channels and uses single mode fiber to transmit the laser light to and from the moving surface.

port circulator. A fiber jumper, which may be up to 100 meters long, connects port \#2 of each circulator to a probe that launches light onto and receives light from the moving surface. The light returning from the probe then re-enters port \#2 of the circulator and exits port \#3 of the circulator, which then directs the light to the detector system. Each detector system consists of a variable fiber attenuator, an optical power meter, and a high-bandwidth detector.

Our heterodyne system is built with four channels to match the number of input channels of the high-bandwidth, highsample-rate digitizers. Each circulator is rated for a maximum $\mathrm{CW}$ capacity of 0.5 watts. The laser was chosen to have a 2watt maximum output so that there is no danger of over-driving the circulators with the $1 \times 4$ fiber splitter. The variable fiber attenuators are used to compensate for any large variations in the amount of light received from the probes. Prior to an experiment, the laser is initially set at a low output level and the return powers are monitored using the optical power meters. The attenuators are used to reduce the amount of light from each probe to the level received by the probe with the weakest return. Once the amount of light entering each detector is nearly equal, the 
laser power is increased until each detector is operating comfortably below saturation levels.

Depending upon the application, the probes may have a wide variety of initial stand-offs from the surface to be measured. We have had initial working distances range from as little as $0.5 \mathrm{~mm}$ to as large as $250 \mathrm{~mm}$ in our various experiments. The probe dimensions are chosen to match the stand-offs in a way so that we generally have collection efficiencies between $1 \times 10^{-4}$ and 0.04 . A good beat signal is usually obtained when the amount of light collected from the surface is approximately equal to the amount of reference light sent to the detector. We use the back-reflection from the fiber endface inside the probe to provide the reference signal. For example, if our probe geometry results in a collection efficiency from the surface of $1 \times 10^{-4}$, we will order probes with a backreflection of $-40 \mathrm{~dB}$. Because the entire system is fiber coupled, the overall system efficiency is very high. This allows the heterodyne system to obtain sufficiently large beat amplitudes with relatively little laser power even though the probe efficiencies may be quite low in some cases.

As mentioned above, the beat frequency is $1.29 \mathrm{GHz}$ for a velocity of $1 \mathrm{~km} / \mathrm{s}$. This means that measuring velocities in the $\mathrm{km} / \mathrm{s}$ range requires a total electronic bandwidth in the multi-GHz range. Our system uses detectors with $12-\mathrm{GHz}$ bandwidth and the digitizers have $8-\mathrm{GHz}$ bandwidth. The composite bandwidth is approximately $6.7 \mathrm{GHz}$, which corresponds to a maximum velocity of over $5 \mathrm{~km} / \mathrm{s}$. For one experiment, we borrowed 18-GHz detectors from David Holtkamp of LANL and a $20-\mathrm{GHz}$ digitizer from Tektronix. With this combination, we were able to measure high-explosive detonation velocities of over $8 \mathrm{~km} / \mathrm{s}$, which yields an apparent velocity of over $12 \mathrm{~km} / \mathrm{s}$.

\section{DATA ANALYSIS}

The data obtained from the heterodyne detectors is a sinusoidal waveform with frequency that varies with the velocity. In our experience, the beat amplitude also varies over a wide range (Figure 2). We presume that the amplitude varies as the amount of light collected from the surface varies during the measurement. There are a number of factors that can affect the light returned to the probe during a measurement, such as surface material, surface roughness, surface tilt, probe efficiency vs. surface position, and spot diameter.

The system diagram (Figure 1) shows that the Doppler-shifted light is returned from the moving surface and the non-dopplershifted light is returned from the fiber endface inside the probe. This basic configuration is actually a Fizeau interferometer (Figure 3). As the moving surface travels a distance $d$, the round-trip distance from the probe to the surface and back to the probe changes by $2 \mathrm{~d}$.

Therefore, when the surface moves a

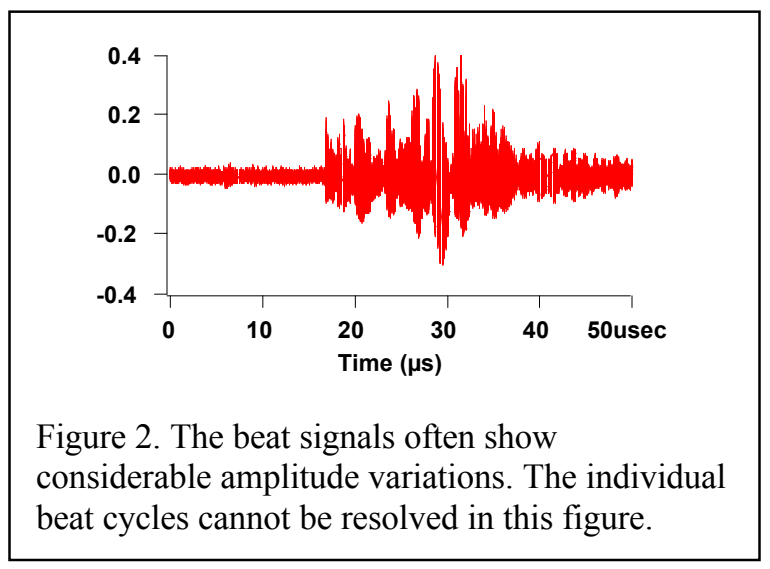




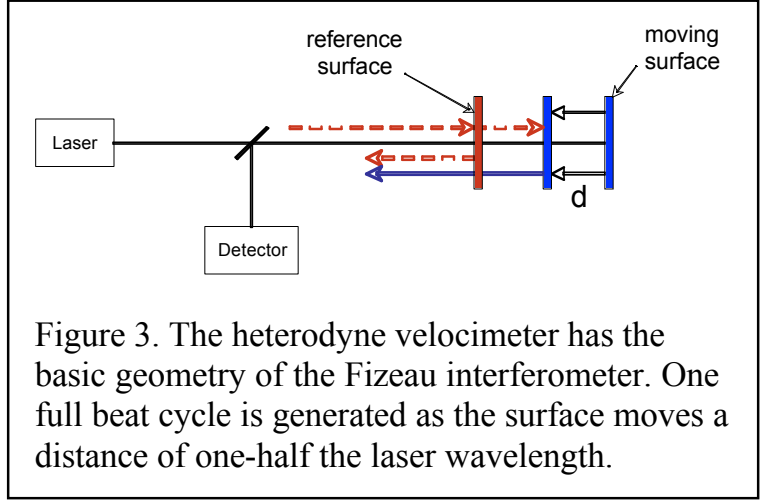

distance equal to one-half the laser wavelength $(\lambda / 2=775 \mathrm{~nm})$, the phase at the probe has changed by a full wavelength. A full beat cycle is created in the data record every time the surface moves $775 \mathrm{~nm}$. The heterodyne velocimeter is actually a distance interferometer.

Knowing that each beat cycle corresponds to a surface motion of $775 \mathrm{~nm}$ suggests one method for analyzing the data. The velocity averaged over one beat cycle is merely $775 \mathrm{~nm}$ divided by the beat period:

$$
v(m / s)=\frac{775}{P(n s)}
$$

If the beat amplitude has enough contrast to accurately determine the location in time of each cycle maximum and minimum (Figure 4), the average velocity over each half cycle may be calculated. For example, at a velocity of $1 \mathrm{~km} / \mathrm{s}$, this method yields one data point every 387 ps. This type of analysis works best for data with many points per cycle, which normally corresponds to low velocities. The data must contain only a single velocity at any time for this method to work.

Most of our data analysis is performed using a sliding Fourier transform method. Bill Kuhlow of NSTec in Las Vegas, NV, (now retired) wrote a code in MatLab that is our workhorse analysis code for most of our data. The user inputs the start time and time per point of the data files, the laser wavelength, and the desired Fourier transform window. The code then calculates the corresponding spectrogram with the horizontal axis equal to the time duration of the input data record, and the vertical axis ranging in frequency from zero to the Nyquist limit. The user is then allowed to select the locus of points in the spectrogram that correspond to the desired velocity profile. This has proven to be a very powerful tool that readily yields the desired velocities even in cases where the beat amplitude signal-to-noise is very low. This method is also able to extract multiple simultaneous velocities from the spectrogram. A typical test geometry for us is to measure at multiple locations the wall velocity of a cylinder filled with highexplosives and detonated at one end. The resulting velocity time histories (Figure 5) were processed with 51-ns Fourier transform windows.

\section{SUMMARY}

Using the heterodyne method to measure velocities in the kilometer-per-second range requires recording frequencies in the



Figure 4. One method to determine the velocity is to measure the time intervals between the minimum and maximum of each beat cycle. 


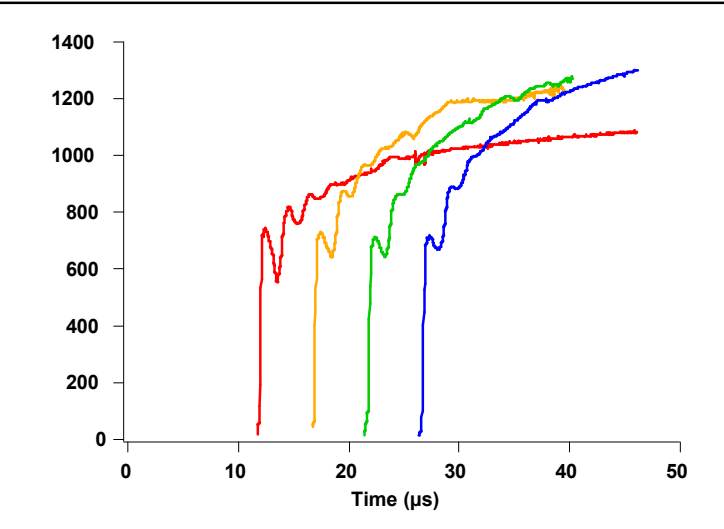

Figure 5. The sliding Fourier transform method of data analysis provides strong clean signals even in cases where the beat amplitude has low signal to noise.

$\mathrm{GHz}$ range. The recent development of high-bandwidth, high-sample-rate digitizers and fiber lasers used by the telecommunications industry has made the assembly of a velocimeter relatively easy using commercially available parts. The system described here operates at a wavelength of $1550 \mathrm{~nm}$ so that a beat frequency of $1.29 \mathrm{GHz}$ corresponds to a velocity of $1 \mathrm{~km} / \mathrm{s}$. The entire system is fiber coupled, which yields very high system efficiencies and renders the system quite safe in terms of laser hazards. The only exposed beam is between the probes and the moving surface. The maximum velocity is determined by the total system bandwidth and the digitizer sample rate. The maximum velocity for the system described here is over $5 \mathrm{~km} / \mathrm{s}$, but using borrowed higher bandwidth components allowed us to measure velocities up to $13 \mathrm{~km} / \mathrm{s}$.

\section{ACKNOWLEDGEMENTS}

This work was performed under the auspices of the U.S. Department of Energy by the University of California, Lawrence Livermore National Laboratory, under contract No. W-7405-Eng-48.

\section{REFERENCES}

1. O.T. Strand, D.R. Goosman, C. Martinez, T.L. Whitworth, W.W. Kuhlow, Rev. Sci. Instrum. 77, 083108 (2006).

2. W.F. Hemsing, Rev. Sci. Instrum. 50, 1 (1979).

3. D.R. Goosman, Appl. Opt. 30, 3907 (1991). 